\title{
Criticality of the random-site Ising model: Metropolis, Swendsen-Wang and Wolff Monte Carlo algorithms
}

\author{
D.Ivaneyko ${ }^{1}$, J.Ilnytskyi ${ }^{2}$, B.Berche ${ }^{3}$, Yu. Holovatch ${ }^{2,4,1}$ \\ 1 Ivan Franko National University of Lviv, \\ 79005 Lviv, Ukraine \\ 2 Institute for Condensed Matter Physics \\ of the National Academy of Sciences of Ukraine, \\ 1 Svientsitskii Str., 79011 Lviv, Ukraine \\ 3 Laboratoire de Physique des Matériaux, \\ Université Henri Poincaré, Nancy 1 , \\ 54506 Vandœuvre les Nancy Cedex, France \\ 4 Institut für Theoretische Physik, \\ Johannes Kepler Universität Linz, \\ 4040 Linz, Austria
}

Received January 12, 2004

\begin{abstract}
We apply numerical simulations to study of the criticality of the $3 D$ Ising model with random site quenched dilution. The emphasis is given to the issues not being discussed in detail before. In particular, we attempt a comparison of different Monte Carlo techniques, discussing regions of their applicability and advantages/disadvantages depending on the aim of a particular simulation set. Moreover, besides evaluation of the critical indices we estimate the universal ratio $\Gamma^{+} / \Gamma^{-}$for the magnetic susceptibility critical amplitudes. Our estimate $\Gamma^{+} / \Gamma^{-}=1.67 \pm 0.15$ is in a good agreement with the recent $\mathrm{MC}$ analysis of the random-bond Ising model giving further support that both random-site and random-bond dilutions lead to the same universality class.
\end{abstract}

Key words: Ising model, quenched disorder, Monte Carlo, cluster algorithms, criticality

PACS: $61.43 . B n, 64.60 . F r, 75.10 . H k$

\section{Introduction}

Since its introduction in the early 1920-ies, the Ising model serves as a paradigm to study criticality of interacting many-particle systems where a single particle can be in two possible states. Such a general problem statement supported in the 1960- 
ies by the universality and scaling hypothesis led to the present situation, when the Ising model is used to explain criticality and scaling in such different fields as ferromagnetism and binary mixtures on the one hand or networks and text series on the other hand. Subsequently, the random-site Ising model is of primary importance in understanding the effect of the structural disorder on criticality. And this explains a lot of theoretical, experimental and computational efforts invested so far in order to shed light on the phenomena occuring therein.

In particular, the three-dimensional (3D) Ising model subject to a weak dilution by non-magnetic impurities changes its universality class [1]. Already this phenomenon called for its explanation and verification. We refer the reader to recent reviews $[2,3]$ where theoretical, experimental and computational data are collected and compared. In our study, we use the Monte Carlo (MC) approach and address the comparison of three different simulational techniques analysing critical behaviour of the 3D quenched diluted Ising model. Some of the outcome is obvious, but we think it is instructive to perform such simulations now, when certain MC tools are sometimes discarded for not obvious reasons. Moreover, a part of our simulations concerns the critical amplitude ratios of the 3D random-site Ising model - a question which was not intensively analysed so far, and where a disagreement between the MC and theoretical predictions exists.

It is our big pleasure and honour to contribute this paper to the Festschrift devoted to Reinhard Folk's 60th birthday: his contribution to the theory of phase transitions in general, and in particular to the problem we consider here, is hard to be overestimated.

\section{Simulation details and results}

The Hamiltonian of the random-site Ising model has the following form

$$
H=-J \sum_{\langle i j\rangle} c_{i} c_{j} S_{i} S_{j}
$$

where $\langle i j\rangle$ denotes the summation over the nearest neighbour sites of 3D simple cubic lattice, $J$ is the interaction constant, $c_{i}=1$ if the $i$-th site is occupied by spin and $c_{i}=0$ otherwise, the Ising spins $S_{i}$ take two values \pm 1 . Occupied sites $\left(c_{i}=1\right)$ are considered to be randomly distributed and quenched in a fixed configuration. For every observable, discussed below, first the Boltzmann average with respect to the spin subsystem is performed for the fixed site configuration, subsequently the averaging over different disorder realizations (configurational average) is performed. We will use the following notations, the number of all sites is $N=L^{3}$ and the number of sites carrying a spin is $N_{p}$. The concentration of spins is defined therefore as $p=N_{p} / N$.

Several separate sets of simulations are performed in this study with the aim to investigate different characteristics of the model. In all the simulations the concentration of spins was taken fixed and equal to $p=0.85$. For the 3D random site Ising model, at spin concentration $p \approx 0.8$, the correction-to-scaling terms appear to be 
particularly small [4]. Therefore, for the concentration chosen we do not take these terms into account in our further analysis. The simulations were performed on a set of the following lattice sizes $L=10,12,16,24,32,48,64,96$ with periodic boundary conditions. The other details, particularly the number of disorder realizations, the simulation lengths and the algorithms employed have been chosen depending on the purpose of particular simulation set.

The purpose of the first set of simulations is to estimate the critical temperature of the model at different $L$. Due to the finite-size scaling theory [5], the finite system of linear size $L$ will demonstrate an evidence of a critical behaviour at a certain temperature $T_{\mathrm{c}}(L)$ which differs from the critical temperature of the infinite system $T_{\mathrm{c}}(\infty)[6]$

$$
T_{\mathrm{c}}(L)=T_{\mathrm{c}}(\infty)+a L^{-1 / \nu}+\cdots,
$$

where the correction-to-scaling terms have been omitted. To find $T_{\mathrm{c}}(L)$ the following procedure was followed. The initial configuration was prepared by randomly scattering the diluted sites on the lattice, and the original orientations of spins were chosen randomly. First, the runs on the smallest lattice size $L=10$ were performed on $10^{2}$ disorder realizations during $5 \cdot 10^{5} \mathrm{MC}$ sweeps using Metropolis algorithm [7]. The preliminary estimate for the critical temperature was taken from the mean-field approximation:

$$
T_{\mathrm{c}}^{\mathrm{MF}}=p \cdot T_{\mathrm{c}}^{\mathrm{pure}},
$$

where $T_{\mathrm{c}}^{\text {pure }}$ is the critical temperature of the pure 3D Ising model [6]. During the simulations the histograms for the potential energy (1) and for the absolute value of the order parameter

$$
M=\frac{1}{N_{p}}\left|\sum_{i=1}^{N} c_{i} S_{i}\right|,
$$

were built and then, using the histogram reweighting technique [8], the temperature region around $T_{\mathrm{c}}^{\mathrm{MF}}$ was explored. For a given disorder realization, the susceptibility was evaluated according to the fluctuational relation

$$
\chi=K N_{p}\left(\left\langle M^{2}\right\rangle-\langle M\rangle^{2}\right),
$$

where $\langle\cdots\rangle$ denotes Boltzmann averaging and $K=\beta J=J / k_{\mathrm{B}} T$ is the dimensionless coupling. The temperatures where $\chi$ has a maximal value were then averaged over all disorder realizations (hereafter, such configurational averaging will be denoted by $\bar{\ldots}$ ) and used as the working estimate for the critical temperature $T_{\text {sim }}(L)$ for $L=10$. This temperature was also used as a preliminary estimate for the critical temperature for the next lattice size $L=12$. Again, for $L=12$ we performed short runs on $10^{2}$ disorder realizations during $5 \cdot 10^{4} \mathrm{MC}$ sweeps. This provides us with the working estimate for the critical temperature for $L=12, T_{\operatorname{sim}}(12)$. The process is continued until all the lattice sizes are processed. The temperatures $T_{\text {sim }}(L)$ obtained in this way have been used for the final simulations of $10^{3}$ disorder realisations from which all the main results are driven. We should note here that one has different possibilities to define the critical temperature of a finite-size system. Along with 
the definition used in this study, one can define $T_{\mathrm{c}}(L)$ as the temperature where the configurational average of $\chi$ has its maximum. However, the same value of $T_{\mathrm{c}}$ is expected in the thermodynamic limit and the same scaling behaviour holds when approaching this limit.

The large clusters of correlated spins exist in the vicinity of the critical point, which in turn leads to the effect of critical slowing down $[9,10]$. As the result, the relaxation time of the system increases dramatically. One can estimate the typical relaxation time during the simulation by observing the (configurationally dependent) autocorrelation function [11] for the potential energy (or, alternatively, for some other characteristic of the system)

$$
C(\mathrm{t})=\frac{\left\langle E\left(\mathrm{t}_{0}\right) E\left(\mathrm{t}_{0}+\mathrm{t}\right)\right\rangle-\left\langle E\left(\mathrm{t}_{0}\right)\right\rangle\left\langle E\left(\mathrm{t}_{0}\right)\right\rangle}{\left\langle E\left(\mathrm{t}_{0}\right) E\left(\mathrm{t}_{0}\right)\right\rangle-\left\langle E\left(\mathrm{t}_{0}\right)\right\rangle\left\langle E\left(\mathrm{t}_{0}\right)\right\rangle}
$$

where $E(\mathrm{t})$ is the instant value for the energy at some effective time $\mathrm{t}$ (in MC simulations $\mathrm{t}$ is measured in $\mathrm{MC}$ sweeps) and $\mathrm{t}_{0}$ is some time origin. The $C(\mathrm{t})$ is averaged over different time origins in the course of simulations. At times large enough, $C(\mathrm{t})$ decays exponentially according to Debye law

$$
C(\mathrm{t})=\exp (-\mathrm{t} / \tau)
$$

and more details can be found elsewhere [12]. In (7) $\tau$ has a meaning of the characteristic autocorrelation time for a given disorder realization. Besides the effect on critical dynamics (which is beyond the scope of this study), the critical slowing down has also some practical implications. Firstly, the estimate of $\tau$ is vital for determination of the required length of the simulation. Only the configurations separated by a number of MC sweeps of order $\tau$ can be considered as statistically independent, and the required length of the simulation run should be measured in $\tau$ scale but not in MC sweeps [12]. Secondly, the critical slowing down leads in Metropolis (or other local dynamics) algorithms to a dramatic increase of $\tau$. This is, of course, due to a high energy penalty required to flip a single spin (or a pair of spins) in a cluster of uniformly oriented neighbours. To overcome this problem, a number of cluster algorithms with non-local dynamics have been suggested, with the Swendsen-Wang [13] and Wolff [14] ones being most commonly used.

Table 1. The energy autocorrelation time $\tau_{\mathrm{E}}$ for different lattice sizes $L$ measured in $\mathrm{MC}$ sweeps (see the text for details).

\begin{tabular}{|l|l|l|l|l|l|l|l|l|}
\hline $\mathrm{L}$ & 10 & 12 & 16 & 24 & 32 & 48 & 64 & 96 \\
\hline$\tau_{\mathrm{E}}$, Metropolis & 5.79 & 7.87 & 12.9 & 26.5 & 44.9 & 93.1 & 160 & 336 \\
\hline$\tau_{\mathrm{E}}$, Swendsen-Wang & 3.55 & 3.98 & 4.69 & 5.54 & 6.10 & 7.50 & 8.55 & 10.7 \\
\hline$\tau_{\mathrm{E}}$, Wolff & 1.18 & 1.24 & 1.31 & 1.46 & 1.54 & 1.71 & 1.81 & 1.98 \\
\hline
\end{tabular}

The second set of our simulations is targeted upon the detailed comparison of autocorrelation times and of the efficiency of different $\mathrm{MC}$ algorithms at a number 
of lattice sizes $L$ for the model under consideration. To this end we performed the simulations of 10-15 disorder realizations for each $L$ at the temperatures $T_{\operatorname{sim}}(L)$. We used three different algorithms, the Metropolis, Swendsen-Wang and Wolff ones. The simulation length was fixed to $10^{5}$ for all cases. For each given disorder realization, the value of $\tau$ has been evaluated according to (7). These values were then averaged over all disorder realizations. The results for the average autocorrelation time $\tau_{\mathrm{E}}$ are presented in table 1 . The relaxation times are given in MC sweeps, where one sweep corresponds to the refreshing of each spin. This means that for the Wolff algorithm we followed the standard procedure of autocorrelation time normalisation taking into account the average cluster size [14]. One can see the different rate of pseudodynamics for all three algorithms used and the autocorrelation time $\tau_{\mathrm{E}}$ can be seen as a measure of the minimal number of MC sweeps separating two adjacent uncorrelated configurations. However, all three algorithms differ significantly by the time spent for one MC sweep and we will be more interested in the time required to generate the next uncorrelated configuration. This will reflect the true speed of each algorithm. The latter can be estimated from an inverse time $t_{\text {trial }}$ spent on $N_{\text {trial }} \mathrm{MC}$ sweeps and taking into account that only $N_{\text {trial }} / \tau_{\mathrm{E}}$ of these are uncorrelated

$$
\lambda=\frac{N_{\text {trial }}}{t_{\text {trial }} \tau_{\mathrm{E}}} .
$$

To this end we performed short trial simulations for 10 disorder realizations each of which was well equilibrated, with $N_{\text {trial }}=10^{3}$. The simulations were performed for all lattice sizes $L=10-96$ and using all three MC algorithms. The results are presented in figure 1 . One can conclude the following. Both cluster algorithms

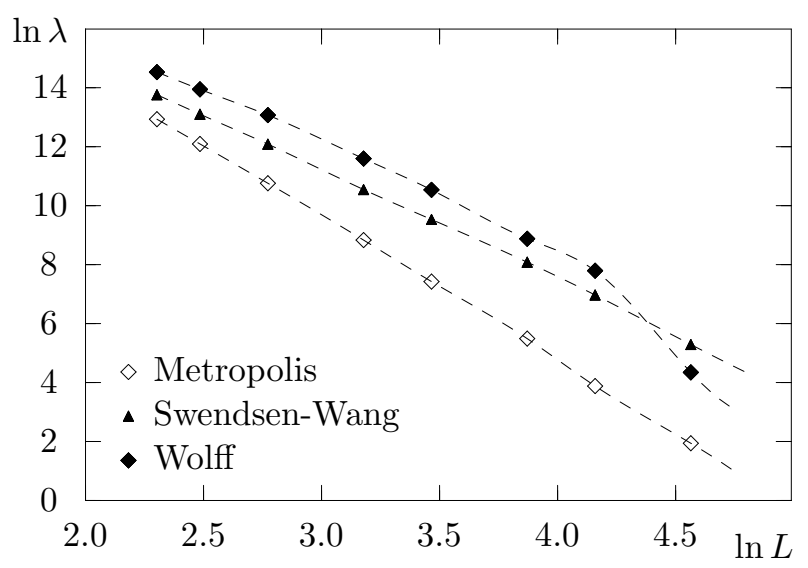

Figure 1. Log-log plot for relative speed measured as by inverse time spent for generating one uncorrelated configuration for lattice sizes $L=10-96$ and three different MC algorithms.

have a significantly higher real speed than the Metropolis one for all lattice sizes. Concerning the larger lattices examined, both cluster algorithms demonstrate comparable speed with the Wolff one being slightly faster for $L<80$. At $L \approx 80$, the 
speed of both cluster algorithms is the same, and for the larger lattice sizes the Swendsen-Wang algorithm looks more preferable (however, we have not done any simulations beyond $L=96$ to check this). According to these findings we can conclude that for the model and lattice sizes examined in this study the Wolff algorithm is the most preferable one and therefore it was used to obtain all the main results in this study. We should also note that our realization of the Metropolis algorithm does not use any special tricks to speed-up the simulation of the Ising model (energy maps, multi-spin coding, etc.). Therefore, our estimate of a true speed of Metropolis algorithm is subject to the particular code being used.

The third set of our simulations is aimed at the evaluation of the critical indices and the extrapolation of the critical temperature $T_{\mathrm{c}}(\infty)$ for the infinite system. Along with the existing MC estimates of the critical indices of diluted 3D Ising model (see e.g. [2,3] for reviews) for the sake of consistency we performed our independent evaluation of these quantities. To this end the MC simulations of $10^{3}$ disorder realizations were performed for lattice sizes $L=10,12,16,24,32,48,64,96$ using Wolff algorithm. The simulation length was chosen equal to $250 \tau_{\mathrm{E}}$ for the equlibration and $10^{3} \tau_{\mathrm{E}}$ for the production runs. These numbers were chosen to balance the errors originated from the limited number of disorder realizations $N_{\text {dis }}$ and the finite length $N_{\mathrm{MC}}$ of the simulations, both terms appearing in the total error estimate for some quantity $A[12]$

$$
\Delta A=\left(\frac{\sigma_{\langle A\rangle}^{2}}{N_{\mathrm{dis}}}+\left(1+2 \tau_{\mathrm{A}}\right) \frac{\sigma_{\mathrm{A}}^{2}}{N_{\mathrm{dis}} N_{\mathrm{MC}}}\right)^{1 / 2}
$$

where

$$
\sigma_{\langle A\rangle}=\left(\overline{\left\langle A^{2}\right\rangle}-\overline{\langle A\rangle}^{2}\right)^{1 / 2}
$$

is the standard deviation of the set of $N_{\text {dis }}$ averages $\langle A\rangle$ calculated within each disorder realizations, and

$$
\sigma_{A}=\left(\overline{\left\langle A^{2}\right\rangle-\langle A\rangle^{2}}\right)^{1 / 2}
$$

is the standard deviation of the whole set of $N_{\text {dis }} N_{\text {MC }}$ data for the quantity $A$. The $\tau_{\mathrm{A}}$ in equation (9) is the integrated autocorrelation time $\tau_{\mathrm{A}}^{\text {int }}[12]$ which, in general differs from the exponentional one $\tau_{\mathrm{A}}^{\exp }$ introduced in (7) in the case of the energy. However, for most of realistic models, $\tau_{\mathrm{A}}^{\text {int }} \leqslant \tau_{\mathrm{A}}^{\exp }[12]$ so that only the use of $\tau_{\mathrm{A}}^{\exp }$ which we employ in this study is reasonable.

In evaluation of the critical indices we followed the standard finite-size scaling scheme [6]. According to it, the Binder's cumulant

$$
U=1-\frac{\left\langle M^{4}\right\rangle}{3\left\langle M^{2}\right\rangle^{2}}
$$

can be evaluated as a function of a coupling $K$ for each disorder configuration and the maximum value for the slope of this function varies with the system size as $L^{1 / \nu}$ anywhere in the critical region. In the terms of temperature derivative one has

$$
\left.\frac{\mathrm{d} U}{\mathrm{~d} K}\right|_{\max }=-\left.T^{2} \frac{\mathrm{d} U}{\mathrm{~d} T}\right|_{\max } \sim a L^{1 / \nu},
$$


where the histogram reweighting technique was used to evaluate the cumulant $U$ in the neighbourhood of $T_{\mathrm{c}}(L)$ and the numerical derivation was employed. Here and thereafter the observables (e. g. (13)-(15)) are averaged over disorder realization. The inverse values for $\nu$ can be found by the linear interpolation of the log-log plot for the values $\left.\overline{\mathrm{d} U / \mathrm{d} K}\right|_{\max }$ vs $L$ (see, figure 2). The same finite size scaling behaviour is valid for the number of logarithmic derivatives for the powers of magnetization [6]. These can be used as additional estimates for the critical index $\nu$, and we evaluated two of them, namely

$$
\begin{aligned}
& \overline{\frac{\mathrm{d}}{\mathrm{d} K} \ln \langle M\rangle}=\overline{-T^{2} \frac{\mathrm{d}}{\mathrm{d} T} \ln \langle M\rangle}, \\
& \overline{\frac{\mathrm{d}}{\mathrm{d} K} \ln \left\langle M^{2}\right\rangle}=\overline{-T^{2} \frac{\mathrm{d}}{\mathrm{d} T} \ln \left\langle M^{2}\right\rangle} .
\end{aligned}
$$

The log-log plots for these derivatives are shown in figure 2, too.

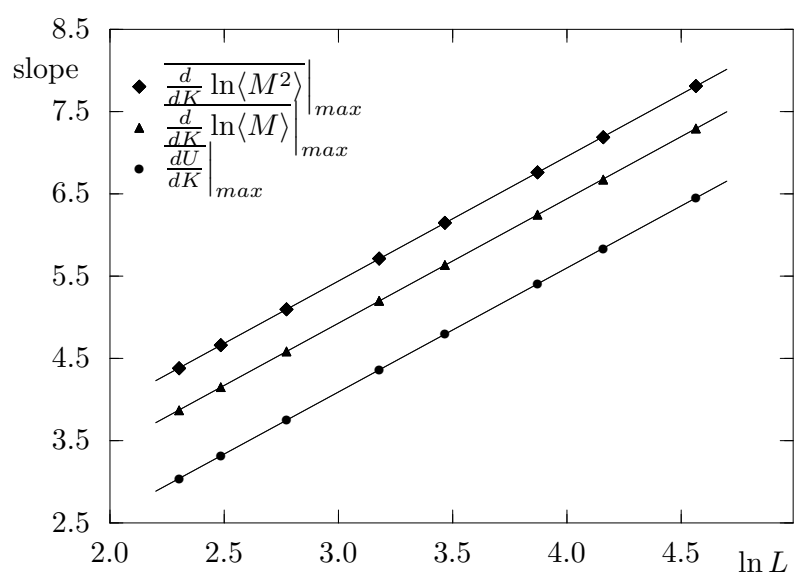

Figure 2. Log-log plots for the maximum values of the configurationally averaged derivatives of Binder cumulant (13) (discs), $\ln \langle M\rangle$ (14) (triangles) and $\ln \left\langle M^{2}\right\rangle$ (15) (diamonds).

First of all we would like to stress that all the data points presented in this figure interpolate extremely well by the appropriate linear dependencies. This can be seen as another proof that the number of disorder realizations and the simulation length that have been used are reasonable. The linear interpolation for the curves presented in figure 2 leads to the values presented in table 2.

The average of all the three results for the index $\nu$ shown in table 2 yields

$$
\nu=0.662 \pm 0.002
$$

The obtained value for $\nu$ distingtly differs from the theoretical estimate of the corresponding exponent of the pure 3D Ising model: $\nu=0.6304(13)$ [19]. However it is smaller than the asymptotic value of $\nu$ for the random 3D Ising model as obtained 
Table 2. The values for the critical index $\nu$ obtained via linear interpolation of the log-log data for the slope of Binder's cumulant and logarithmic derivatives of $\langle M\rangle$ and $\left\langle M^{2}\right\rangle$

\begin{tabular}{|c|c|c|}
\hline interpolation of & $1 / \nu$ & $\nu$ \\
\hline$\left.\overline{\overline{\mathrm{d} U}}\right|_{\max }$ & $1.507 \pm 0.008$ & $0.664 \pm 0.004$ \\
\hline$\left.\overline{\frac{\mathrm{d}}{\mathrm{d} K} \ln \langle M\rangle}\right|_{\max }$ & $1.512 \pm 0.001$ & $0.661 \pm 0.001$ \\
\hline$\left.\overline{\frac{\mathrm{d}}{\mathrm{d} K} \ln \left\langle M^{2}\right\rangle}\right|_{\max }$ & $1.514 \pm 0.001$ & $0.660 \pm 0.001$ \\
\hline
\end{tabular}

by the field-theoretical RG approach $\nu=0.678(10)[20]$ and by the MC simulations $\nu=0.6837(53)[4], \nu=0.683(3)$ [18]. This is an evidence of the fact that for the spin concentrations and lattice sizes chosen here the system still crosses over to the asymptotic regime. Note that close estimates for $\nu$ were found at similar system parameters (and neglecting correction-to-scaling terms) in the MC simulation of the random site 3D Ising model: $p=0.9, L=64 \div 128, \nu=0.6644(15)$ [4]; as well as of the random bond 3D Ising model: $p_{\text {bonds }}=0.7, \nu=0.660(10)[16]$.

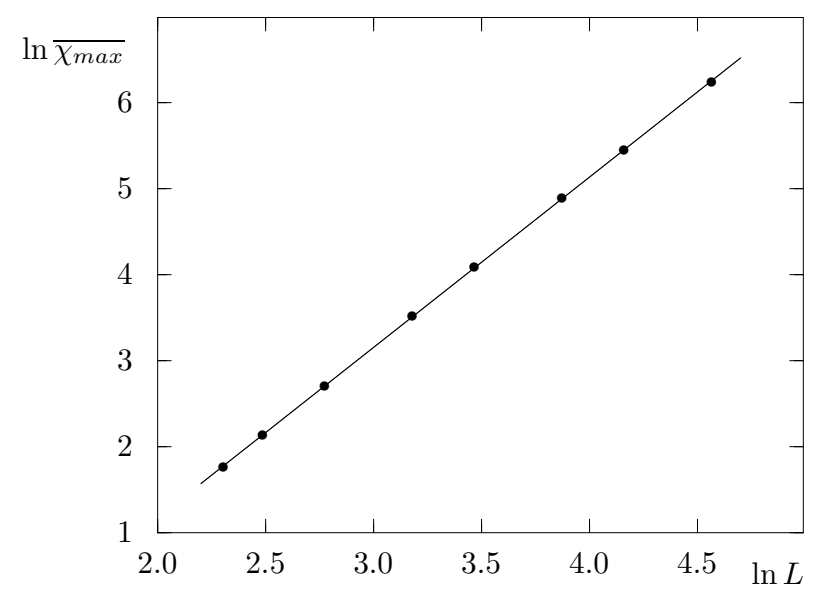

Figure 3. Log-log plot for the maximum value of the susceptibility $\overline{\chi_{\max }}$ vs the linear size of the system $L$ in the critical region.

The standard finite-size scaling concepts have been also employed for the evaluation of the other critical indices, particularly those for the susceptibility $\gamma$ and for the magnetization $\beta$. Due to this scheme [6] for finite size system of dimension $L$ in the critical region one has

$$
\overline{\chi_{\max }} \sim \Gamma L^{\gamma / \nu}, \quad \overline{\langle M\rangle} \sim B L^{-\beta / \nu},
$$

where $\Gamma$ and $B$ are the critical amplitudes and the correction-to-scaling terms have 
been omitted. In (17) $\overline{\chi_{\max }}$ is the averaged maximum value for the susceptibility that is achieved at some temperature and $\overline{\langle M\rangle}$ is the averaged value of the magnetization at the same temperature. We employed the following scheme. For each disorder realization the temperature $T^{*}$ was found where the $\chi$ reaches its maximal value $\chi_{\max }$. Then, the magnetization $\langle M\rangle$ is evaluated at the same $T^{*}$. Afterwards, the values of $\chi_{\max }$ and $\langle M\rangle$ have been averaged over all disorder realizations to be used for fitting the relations (17). The results of fittings are presented in figures 3,4 in a form of the log-log plots for the $\overline{\chi_{\max }}$ and for $\overline{\langle M\rangle}$ vs $L$, respectively.

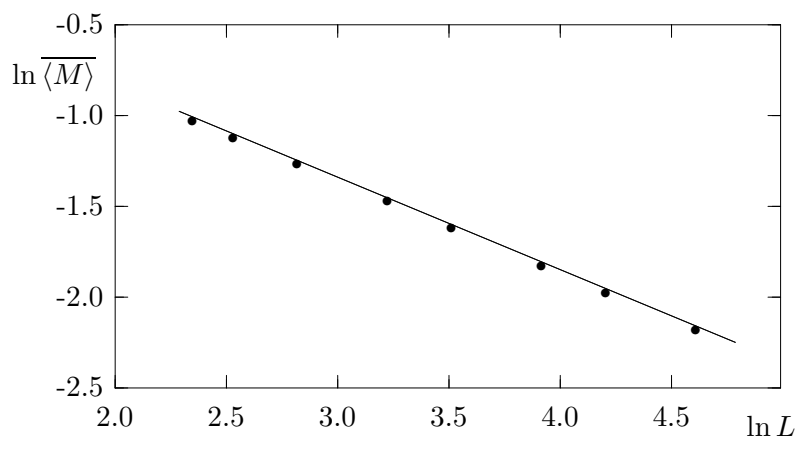

Figure 4. $\log -\log$ plot for the magnetization $\overline{\langle M\rangle}$ vs the linear size of the system $L$ in the critical region.

One should note the near perfect linear fits achieved in both cases, leading to the following results for $\gamma$ and $\beta$

$$
\begin{aligned}
\gamma / \nu & =1.986 \pm 0.001 \\
\beta / \nu & =0.509 \pm 0.001 \\
\gamma & =1.314 \pm 0.004 \\
\beta & =0.337 \pm 0.001
\end{aligned}
$$

For the reasons explained above, taking exponent $\nu$ as an example, the values (18)(21) slightly differ from their asymptotic counterparts $\beta=0.3546(28), \gamma=1.342(10)$ [4]. They are in a good agreement with the exponents of the 3D Ising model with random bond dilution: $\beta / \nu=0.515(5), \gamma / \nu=1.97(2)[16]$ giving one more proof that both models belong to the same universality class.

With the value for the critical index $\nu$ being evaluated in (16) one can obtain the estimate for the critical temperature for the infinite system $T_{\mathrm{c}}(\infty)$. It is related to the critical temperatures of the finite system of linear dimension $L, T_{\mathrm{c}}(L)$, via the relation (2). The values for $T_{\mathrm{c}}(L)$ were estimated during the same set of simulations that have been used for the evaluation of critical indices. For each disorder realization we found the sets of temperatures $T_{\mathrm{c}}^{i}(L)$ where the maximum values for the susceptibility (for the $i=1$ case) and for the derivatives (13)-(15) (the cases $i=2,3,4$, respectively) are achieved. The data sets $T_{\mathrm{c}}^{i}(L)$ should be plotted then vs the scaled system size $L^{-1 / \nu}$ and, ideally, for all $i$ should extrapolate at $L \rightarrow \infty$ to 
the same value of $T_{\mathrm{c}}(\infty)$. The results are shown in figure 5 where we used the data for all the lattice sizes from $L=10$ to $L=96$. As the result of the extrapolation procedure we obtain

$$
T_{\mathrm{c}}^{i}(\infty)= \begin{cases}3.756931 \pm 0.000058, & \left.\chi\right|_{\max } \\ 3.756783 \pm 0.000243, & \mathrm{~d} U /\left.\mathrm{d} K\right|_{\max } \\ 3.756481 \pm 0.000086, & \mathrm{~d} \ln \langle M\rangle /\left.\mathrm{d} K\right|_{\max } \\ 3.756543 \pm 0.000083, & \mathrm{~d} \ln \left\langle M^{2}\right\rangle /\left.\mathrm{d} K\right|_{\max }\end{cases}
$$

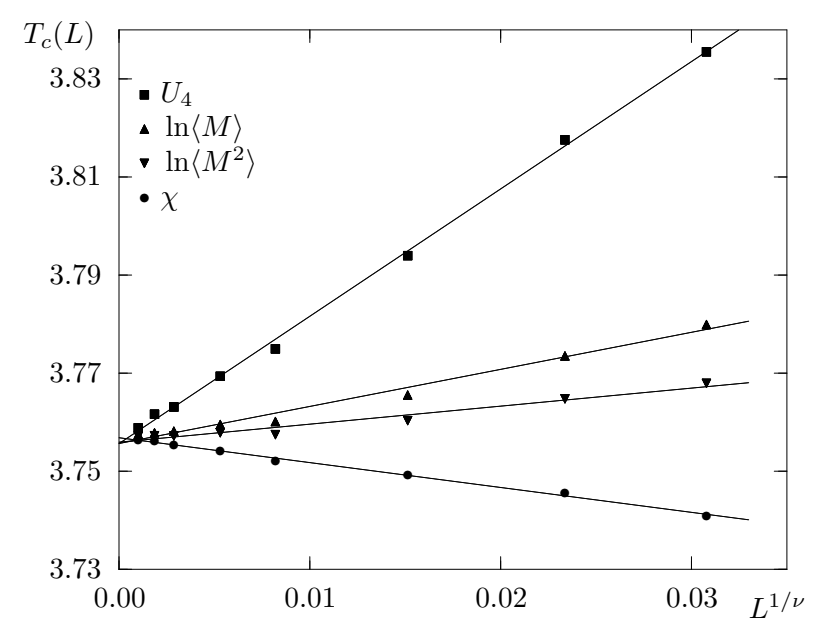

Figure 5. The linear fits for the critical temperatures $T_{\mathrm{c}}^{i}(L)$ vs the scaled system size $L^{-1 / \nu}$ for the lattice sizes from $L=10$ to $L=96$, the index $i$ corresponds to the maximum values for different quantities, see notations in the figure.

One should note a very good convergence of the extrapolated values for $T_{\mathrm{c}}^{i}(\infty)$ found by examining different quantities and given in equation (22). The final estimate for the critical temperature of the infinite system $T_{\mathrm{c}}(\infty)$ can be found as an average on all four numbers in equation (22)

$$
T_{\mathrm{c}}(\infty)=3.7566845 \pm 0.0001175, \quad \beta_{\mathrm{c}}=T_{\mathrm{c}}^{-1}(\infty)=0.2661922 \pm 0.0000083 .
$$

As it is clear already from simple mean-field arguments (see equation (3)) our estimate for $T_{\mathrm{c}}$ made for the spin concentration $p=0.85$ should lay in between the corresponding data for $p=0.8$ and $p=0.9$. Indeed, the most recent estimates read: $\beta_{\mathrm{c}}(p=0.8)=0.2857368(52) ; \beta_{\mathrm{c}}(p=0.9)=0.2492880(35)[4], \beta_{\mathrm{c}}(p=0.8)=$ $0.2857447(24)[18]$.

In this study we have calculated the universal ratio $\Gamma^{+} / \Gamma^{-}$for the magnetic susceptibility in the critical region

$$
\bar{\chi}= \begin{cases}\Gamma^{+} t^{-\gamma}, & T>T_{\mathrm{c}} \\ \Gamma^{-} t^{-\gamma}, & T<T_{\mathrm{c}} .\end{cases}
$$

The singularity of the susceptibility (24) is observed for the infinite system only, at finite system size $L$, it is rounded-off with the finite maximum value. At each given 
system size $L$ there will be only a finite temperature interval where the susceptibility curve overlaps with that for the infinite system. As $T$ approaches the $T_{\mathrm{c}}(\infty)$, the finite-size curve deviates from the infinite one. As a result, each estimate for the critical amplitudes made at different system sizes will be valid in a certain temperature range. The other complication for the disordered systems is the presence of some distribution of $\chi$ curves obtained for different disorder realizations (the example for the system size $L=48$ is presented in figure 6). We employed the following method

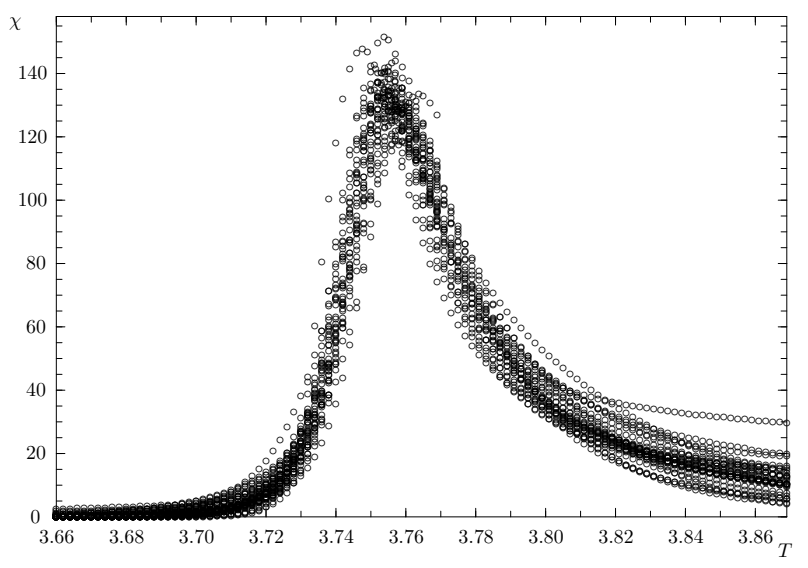

Figure 6. The example of the distribution for the susceptibility vs temperature curves for different disorder realizations for the system size $L=48$.

for the evaluation of critical amplitudes $\Gamma^{+}$and $\Gamma^{-}$. The critical temperature for the infinite system $T_{\mathrm{c}}(\infty)$ was taken as the central one and then the grid of temperatures around it was considered. The gridness was chosen to be even in scaled temperature units $L^{1 / \nu}|t|$, where $t=K_{\mathrm{c}}(\infty)-K=\left[T-T_{\mathrm{c}}(\infty)\right] /\left[T T_{\mathrm{c}}(\infty)\right]$. At each grid temperature the separate simulation of $10^{2}$ disorder realizations was performed of the length $10^{3} \tau_{\mathrm{E}}$ and the value for the susceptibility was evaluated. One could, in principle, use the histogram reweighting technique to evaluate the intermediate temperatures

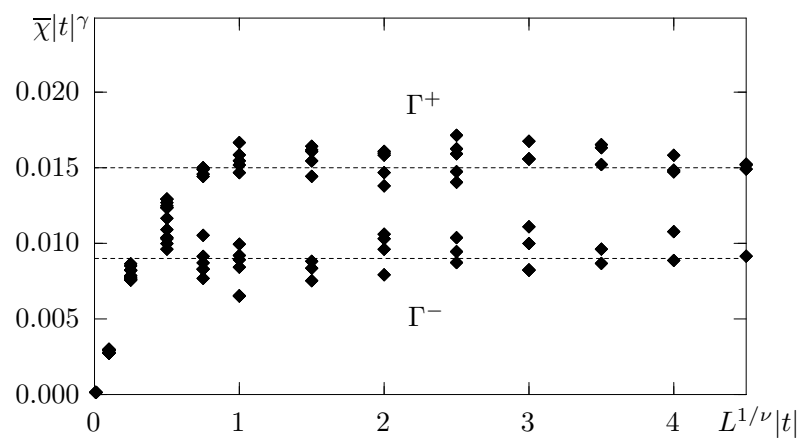

Figure 7. The values for the critical amplitudes for the susceptibility $\Gamma^{+}$and $\Gamma^{-}$ at $T>T_{\mathrm{c}}(\infty)$ and $T<T_{\mathrm{c}}(\infty)$, respectively. 
as well, but this will require the study of histogram validity. In this calculation we opted for more straightforward approach using the separate simulation runs and did not employ histogram reweighting.

The values for the critical amplitudes $\Gamma^{+}$and $\Gamma^{-}$can be obtained by plotting the scaled susceptibility $\bar{\chi}|t|^{\gamma}$. The data are presented in figure 7 and for the evaluation of the ratio $\Gamma^{+} / \Gamma^{-}$the data points with $L^{1 / \nu}|t|<1$ have been ignored, since they are in the FSS regime. This gives the following value for the amplitude ratio

$$
\Gamma^{+} / \Gamma^{-}=1.67 \pm 0.15
$$

It compares very well with the recent result $\Gamma^{+} / \Gamma^{-}=1.62 \pm 0.1$ [16] obtained by MC simulations of bond-diluted Ising model at bond dilution $p_{\text {bond }}=0.7$. The theoretical prediction obtained by the field-theoretical renormalization group calculations in three-loop approximation reads: $\Gamma^{+} / \Gamma^{-}=3.05(32)[17]$. As a reason of the discrepancy between estimates (25), [16] and [17] one can mention the shortness and bad convergence of the series for $\Gamma^{+} / \Gamma^{-}$, as recognized already in [17]. However further analysis is required to get a definite answer. In any case, both theoretical and MC estimates of the critical amplitudes ratio certainly differ from those of the pure 3D Ising model: $\Gamma^{+} / \Gamma^{-}=4.70 \div 4.95$ (see $[21]$ and references therein).

\section{Conclusions and outlook}

In this study we performed computer simulations of the 3D Ising model with random site dilution in critical region. Despite the number of simulation studies of this model being already performed, some aspects of its critical behaviour and simulational details are still awaiting for more detailed analysis. One of these to mention is the applicability and the effectiveness of different Monte Carlo algorithms, the topic previously studied for the pure models mainly. However in the case of disordered models the impurities may have a profound effect on system clusterization which, in turn, is exploited in cluster methods.

We examined the most commonly used Metropolis, Swendsen-Wang and Wolff algorithms and evaluated both the energy autocorrelation times and the respective speed of algorithms in generating statistically independent configurations. We found that for the linear lattice sizes up to $L=100$ the Wolff algorithm is more preferable to use and hence it was applied in our study.

We used the workstations cluster of the ICMP currently equipped by the Athlon MP-2200+ processors. The typical simulation times range from 0.1 seconds per $1000 \mathrm{MC}$ sweeps for the smallest lattice size $L=10$ (Wolff algorithm) to about 953 seconds for the largest lattice size $L=96$ (Metropolis algorithm) per one disorder realization.

Following the standard ideas of the finite size scaling, the critical indices have been calculated. In this way we complemented the existing results. Based on the fact that the correction-to-scaling terms appear to be particularly small [4] in the region of spin concentration $p \approx 0.8$, we have performed the simulation for the concentration $p=0.85$ only. The obtained values for the critical exponents (16), (20), 
(21) slightly differ from their asymptotic values as obtained by the field-theoretical RG approach [20] and by recent MC simulations [4], [18]. This is the consequence of the fact that for the spin concentrations and lattice sizes chosen here the system still crosses over to the asymptotic regime.

Contrary to the critical indices, the values of critical amplitude ratios have received less attention so far. In our study, we get an estimate for the magnetic susceptibility critical amplitude ratio $\Gamma^{+} / \Gamma^{-}=1.67 \pm 0.15$. This value is in a good agreement with the recent MC analysis of the random-bond Ising model [16] giving further support that both random-site and random-bond dilutions lead to the same universality class.

The comparative analysis of different MC algorithms (including Metropolis, Swendsen-Wang and Wolff ones) which is a subject of this study will be used in our future work regarding the effect of different types of structural disorder on criticality.

\section{Acknowledgements}

We acknowledge useful discussions with Wolfhard Janke and Christophe Chatelain. This work was supported by the French-Ukrainian cooperation "Dnipro" programme. Work of Yu.H. was supported in part by the Austrian Fonds zur Förderung der wissenschaftlichen Forschung, project No. 16574-PHY.

It is our special pleasure and pride to acknowledge wonderful hospitality and high spirit of people in Kyiv, where several of the authors met in December days of 2004 and where a part of this paper was finalized.

\section{References}

1. Harris A.B., J. Phys. C, 1974, 7, 1671.

2. Pelissetto A., Vicari E., Phys. Rep, 2002, 368, 549.

3. Folk R., Holovatch Y., Yavors'kii T., Uspekhi Fiz. Nauk, 2003, 173, 175.; Phys. Usp., 2003, 46, 175.

4. Ballesteros H.G., Fernández L.A., Martiń-Mayor V., Muñoz Sudure A., Phys. Rev. B, 1998, 58, 2740.

5. Barber M.N. Finite-size scaling. Phase Transitions and Critical Phenomena, eds. Domb C. and Lebowitz J., vol. 8. Academic, New York, 1983.

6. Ferrenberg A.M., Landau D.P., Phys. Rev. B, 1991, 44, No. 10, 5081-5091.

7. Metropolis N. et al, J. Chem. Phys., 1953, 21, No. 6, 1087-1092.

8. Ferrenberg A.M., Swendsen R.H., Phys. Rev. Lett., 1988, vol. 61, No. 23, 2635-2638.

9. Hohenberg P.C., Halperin B.I., Rev. Mod. Phys., 1977, 49, No. 3, 435-479.

10. Sokal A.D. MC Methods in Statistical Mechanics: Foundations and New Algorithms, Cours de Troisiéme Cycle de la Physique en Suisse Romande. Lausanne, June 1989.

11. Allen M.P., Tildesley D.J. Computer Simulation of Liquids. Clarendon Press, Oxford, 1987.

12. Janke W. Statistical analysis of simulations: data correlations and error estimation. Quantum Simulations of Complex Many-Body Systems: From Theory to Algorithms, Lecture Notes, eds. Grotendorst J., Marx D., Muramatsu A, vol. 10, p. 423-445. John von Neumann Institute for Computing. Jülich, NIC Series, 2002. 
13. Swendsen R.H., Wang J.-S., Phys. Rev. Lett., 1987, 58, No. 2, 86-88.

14. Wolff U., Phys. Rev. Lett., 1989, 62, No. 4, 361-364.

15. Wang J.-S., Kozan O., Swendsen R.H., Phys. Rev. E, 2002, 66, 057101.

16. Berche P.E., Chatelain C., Berche B., Janke W., Eur. Phys. J. B, 2004, 38, 463.

17. Bervillier C., Shpot M., Phys. Rev. B, 1992, 46, No. 2, 955-968.

18. Calabrese P., Martiń-Mayor V., Pelissetto A., Vicari E., Phys. Rev. B, 2003, 68, 036136 .

19. Guida R., Zinn-Justin J., J. Phys. A, 1998, 31, 8103.

20. Pelissetto A., Vicari E., Phys. Rev. B, 2000, 62, 6393.

21. Caselle M., Hasenbusch M., J. Phys. A, 1997, 30, 4963.

\title{
Критична поведінка моделі Ізинга з випадковими вузлами: Монте Карло алгоритми Метрополіса, Свендсен-Ванга та Вольфа
}

\author{
Д.Іванейко ${ }^{1}$, Я.Ільницький ${ }^{2}$, Б.Берш ${ }^{3}$, Ю.Головач ${ }^{2,4,1}$ \\ 1 Львівський національний університет ім. І.Франка, \\ 79005 Львів, Україна \\ 2 Інститут фізики конденсованих систем \\ Національна академія наук України, \\ вул. Свєнціцького 1, 79011 Львів, Україна \\ 3 Лабораторія фізики матеріалів, \\ Університет ім. Анрі Пуанкаре 1, \\ 54506 Вандувр лє Нансі Седекс, Франція \\ 4 Інтитут теоретичної фізики, \\ Університет Йогана Кеплера, \\ 4040 Лінц, Австрія
}

Отримано 12 січня 2004 р.

Ми застосовуємо комп'ютерні симуляції для вивчення критичної поведінки тривимірної моделі Ізинга з випадковим вузловим замороженим розведенням. Акцент зроблений на тому, що досі ще детально не обговорювалось. Зокрема, ми намагаємося порівняти різні техніки Монте Карло, обговорюючи області їхнього застосування та їхні переваги/недоліки, в залежості від мети досліджень. Також, крім обчислення критичних індексів, ми оцінили універсальне відношення $\Gamma^{+} / \Gamma^{-}$для критичних амплітуд магнітної сприйнятливості. Наша оцінка $\Gamma^{+} / \Gamma^{-}=1.67 \pm 0.15$ добре узгоджується $з$ недавно отриманим результатом комп'ютерних симуляцій моделі Ізинга з випадковими зв'язками, тим самим підтверджуючи, що моделі Ізинга з випадковими зв'язками і випадковими вузлами належать до одного класу універсальності.

Ключові слова: модель Ізинга, заморожений безлад, Монте Карло, кластерні алгоритми, критичність

PACS: $61.43 . \mathrm{Bn}, 64.60 . \mathrm{Fr}, 75.10 . \mathrm{Hk}$ 